\title{
Synchronization of neural networks with stochastic perturbation via aperiodically intermittent control
}

\author{
Wei Zhang*a ${ }^{* a}$ Chuandong $\mathrm{Li}^{\mathrm{a}}$, Tingwen Huang ${ }^{\mathrm{b}}$, Mingqing Xiao ${ }^{\mathrm{c}}$ \\ ${ }^{a}$ College of Computer Science, Chongqing University, Chongqing 400044, PR China \\ ${ }^{b}$ Department of Mathematics, Texas AEGM University at Qatar, Doha, P.O.Box 23874, Qatar \\ ${ }^{c}$ Department of Mathematics, Southern Illinois University, IL 62901, USA
}

\begin{abstract}
In this paper, the synchronization problem for neural networks with stochastic perturbation is studied with intermittent control via adaptive aperiodicity. Under the framework of stochastic theory and Lyapunov stability method, we develop some techniques of intermittent control with adaptive aperiodicity to achieve the synchronization of a class of neural networks, modeled by stochastic systems. Some effective sufficient conditions are established for the realization of synchronization of the underlying network. Numerical simulations of two examples are provided to illustrate the theoretical results obtained in the paper.
\end{abstract}

Keywords: synchronization, neural networks, stochastic perturbation, aperiodically intermittent control, adaptive control;

\section{Introduction}

Complex networks are playing more and more important roles in our today's society. A complex network is characterized by a large set of nodes that are connected through a set of links for the communication and interaction purpose. Many real world phenomena, such as those appeared in telecommunication, biological formation, chemical reaction, neural networks, social organization, the World Wide Web, ... etc., can be described or modeled by complex networks. Since the seminal papers that started to discuss the "small-world" and "scale-free" properties [1][2], the study of complex networks is not only becoming one of the main research areas in the network society but also brought a great of attention from researchers of different fields. With the viewpoint of complex dynamical networks, many interesting and important dynamical behaviors, such as synchronization, consensus, self-organization, combinatorial optimization, and spatiotemporal chaos of spiral waves, have been studied [3]-[14]. Over the past decades, synchronization of large-scale complex networks consisting of coupling dynamical systems has been extensively investigated in various disciplines [13]-[16].

As a special class of complex networks, coupled neural networks have been a hot topic because they have wide applications in a variety of areas. One of the focus topics in the investigation of neural networks is the synchronization of all dynamical nodes in a network, resulting from its important applications such as in image processing, general neural networks, secured communication and network updating. Meanwhile, many control methodology has been developed in order to synchronize neural networks governed by nonlinear systems, such as adaptive control [18], fuzzy control [19], impulsive control [20]-[23] and intermittent control [24].

Intermittent control, which was first introduced to control the nonlinear dynamical systems in [25], has been used for a variety of purposes such as manufacturing, transportational and communication. In the past, the intermittent control was mainly periodically intermittent control [26]-[29]. In [26], periodically intermittent control is used for the neural networks with time-varying delays to a desired orbit. In [27], the authors have discussed the exponential lay synchronization for delayed fuzzy cellular neural networks via periodically intermittent control. Meanwhile, uncertainties always exist in the real applications, such as stochastic forces on the physical systems and noisy measurements caused by environment complexity. For instance, signals transmitted between nodes of neural networks are unavoidably subject to stochastic perturbations from

\footnotetext{
*Corresponding author

Email addresses: cquzw@hotmail.com (Wei Zhang*), licd@cqu .edu.cn (Chuandong Li), tingwen.huang@qatar . tamu.edu (Tingwen Huang), mxiao@siu.edu (Mingqing Xiao)
} 
environment, which may cause information contained in these signals being lost. Therefore, stochastic perturbations can not be ignored in gerenal [29]-[33]. In [29], the exponential synchronization of stochastic perturbed complex networks with time-varying delays via periodically intermittent pinning was studied. In [32], stochastic synchronization of coupled neural networks with intermittent control was also investigated.

The requirement of periodicity of intermittent control strategy may not be suitable in reality. For example, the generation of wind power in smart grid relies on the various situations of our real world, which is obviously aperiodically intermittent. Therefore, for the theoretical analysis of real applications, it is more practical to consider the synchronization problem under aperiodically intermittent control strategy. To the best of our knowledge, there have been few results for the study of dynamical behaviors in terms of aperiodically intermittent control strategy. In [34], synchronization of nonlinear coupled networks via aperiodically intermittent pinning control was investigated. In this paper, we will study the synchronization of nonlinear coupled complex networks with stochastic perturbation via aperiodically intermittent control.

Motivated by above discussions, in this paper, we will investigate the problem of synchronization of neural networks with stochastic perturbation via aperiodically intermittent control. Firstly, we will establish sufficient conditions for nonlinear coupled networks under aperiodically intermittent control to achieve synchronization. Secondly, by virtue of properties of Weiner process and estimation techniques, suitable aperiodically intermittent and adaptive aperiodically intermittent controllers are designed to ensure stochastic synchronization for the coupled complex networks with stochastic perturbations. Synchronization criteria obtained in the paper are simple and verifiable, and hence it is practically useful in applications. The obtained theoretical results will be illustrated by numerical simulations in last section.

The structure of this paper is organized as follows: in Section 2, we will introduce the neural network model with stochastic perturbation in terms of aperiodically intermittent control as well as some notations. Sufficient synchronization conditions with mathematical justifications are presented in Section 3. Two illustrative example are given to demonstrate the effectiveness of the proposed approach in Section 4. The paper ends with concluding remarks in Section 5.

Notations: The following notations will be used throughout this paper. $\lambda_{\max }(\cdot)$ stands for the maximum eigenvalue of a real matrix. $\mathbb{R}^{+}$and $\mathbb{R}^{n}$ represent, respectively, the set of nonnegative real numbers and the n-dimensional Euclidean space. $\mathbb{R}^{n \times n}$ is used for the set of all $n \times n$ real matrices. $\|\cdot\|$ is the standard Euclidean norm in $\mathbb{R}^{n} . A=\left(a_{i j}\right)_{n \times n}$ stands for an $n \times n$ matrix with entries $a_{i j}$. The superscript $T$ denotes the transpose of a matrix or a vector. $I_{n}$ is the $n \times n$ identity matrix.

\section{Problem formulation and some preliminaries}

We consider a neural network system consisting of $N$ identical nodes that are nonlinear coupling with vector-form stochastic perturbations, which is described by

$$
\dot{x}_{i}(t)=\left[-C x_{i}(t)+B f\left(x_{i}(t)\right)+\sum_{j=1, i \neq j}^{N} a_{i j}\left[\phi_{j}\left(x_{j}(t)\right)-\phi_{i}\left(x_{i}(t)\right)\right]\right] d t+\sigma\left(x_{i}(t)\right) d \omega(t)
$$

where $x_{i}(t)=\left(x_{i 1}(t), x_{i 2}(t), \ldots, x_{i n}(t)\right)^{T} \in \mathbb{R}^{n}$ represents the state vector of the $i$ th node of the network; $C=\operatorname{diag}\left(c_{1}, c_{2}, \ldots, c_{n}\right)$ with $c_{k}>0, k=1,2, \ldots, n$, denotes the rate with which the $k$ th cell rests its potential to the resting state when isolated from other cells and inputs; $B=\left[b_{i j}\right]_{n \times n} \in \mathbb{R}^{n \times n}$ represent the connection weight matrix; $A=\left[a_{i j}\right]_{n \times n} \in \mathbb{R}^{N \times N} ; f\left(x_{i}(t)\right)=$ $\left[f_{1}\left(x_{i}(t)\right), f_{2}\left(x_{i}(t)\right), \ldots, f_{n}\left(x_{i}(t)\right)\right]^{T}$ is a continuous vector; $\sigma\left(x_{i}(t)\right)=\sigma\left(x_{1}, x_{2}, \ldots, x_{n}\right) \in \mathbb{R}^{n \times n}$ is the noise intensity matrix and $\omega(t)=\left(\omega_{1}(t), \omega_{2}(t), \ldots, \omega_{n}(t)\right)^{T} \in \mathbb{R}^{n}$ is bounded vector-form Weiner process, satisfying $E \omega_{j}(t)=0, E \omega_{i}^{2}=1, E \omega_{j}(t) \omega_{j}(s)=$ $0(s \neq t)$.

In the case that system (1) reaches synchronization, i.e. $x_{1}(t)=x_{2}(t)=\cdots=x_{N}(t)=s(t)$, by introducing a controller into each individual node, where $s(t) \in \mathbb{R}$ is defined as

$$
\dot{s}(t)=[-C s(t)+B f(s(t))] d t+\sigma(s(t)) d \omega(t)
$$

and $s(t)$ can be set to be any desired state: either equilibrium point, or a nontrivial periodic orbit, or even a chaotic orbit.

In order to achieve the synchronization objective, the aperiodically intermittent controllers will be applied to some of its nodes. For the convenience of description, we denote $\phi\left(x_{j}(t), x_{i}(t)\right)=\phi_{j}\left(x_{j}(t)\right)-\phi_{i}\left(x_{i}(t)\right)$. Thus the intermittent controlled network can be formulated as

$$
\dot{x}_{i}(t)=\left[-C x_{i}(t)+B f\left(x_{i}(t)\right)+\sum_{j=1, i \neq j}^{N} a_{i j} \phi\left(x_{j}(t), x_{i}(t)\right)+u_{i}(t)\right] d t+\sigma\left(x_{i}(t)\right) d \omega(t)
$$


where $u_{i}(t)(i=1,2, \ldots, n)$ are the intermittent linear state feedback controller and it is constructed as following

$$
u_{i}(t)= \begin{cases}-\varepsilon_{i} \phi\left(x_{i}(t), s(t)\right), & t \in\left[t_{i}, s_{i}\right), \\ 0, & t \in\left[s_{i}, t_{i+1}\right), i=0,1,2, \ldots\end{cases}
$$

where $\varepsilon_{i}>0$ represents control gain and $\Xi=\operatorname{diag}\left(\varepsilon_{1}, \varepsilon_{2}, \ldots, \varepsilon_{N}\right) \in \mathbb{R}^{n \times n}$. The synchronization error is defined to be $e_{i}(t)=x_{i}(t)-s(t)$. By the controller expression (4), the error dynamics is governed by

$$
\dot{e}_{i}(t)=\left[-C e_{i}(t)+B g\left(e_{i}(t)\right)+\sum_{j=1, i \neq j}^{N} a_{i j} \phi\left(x_{j}(t), x_{i}(t)\right)+u_{i}(t)\right] d t+\tilde{\sigma}\left(e_{i}(t)\right) d \omega(t)
$$

where $g\left(e_{i}(t)\right)=f\left(x_{i}(t)\right)-f(s(t))$ and $\tilde{\sigma}_{i}(t)=\sigma\left(x_{i}(t)\right)-\sigma(s(t))$.

Assumption 1: For the aperiodically intermittent control strategy, there exist two positive scalar $0<\theta<\omega$, such that, for $i=0,1,2$

$$
\left\{\begin{array}{l}
\inf _{i}\left(s_{i}-t_{i}\right)=\theta>0 \\
\sup _{i}\left(t_{i+1}-t_{i}\right)=\omega<+\infty
\end{array}\right.
$$

Assumption 2: In the following, we assume that the nonlinear coupling function (or protocol) has the following form:

$$
\phi_{j}\left(x_{1}, x_{2}, \ldots, x_{N}\right)=\sum_{k=1, k \neq j}^{N} a_{j k} \phi\left(x_{k}, x_{j}\right)
$$

where $a_{j k} \geq 0$, and function $\phi(\cdot, \cdot): \mathbb{R}^{n} \times \mathbb{R}^{n} \rightarrow \mathbb{R}^{n}$ has the form

$$
\Phi(X, Y)=\left(\phi\left(x_{1}, y_{1}\right), \ldots, \phi\left(x_{n}, y_{n}\right)\right)^{T}
$$

for any $X=\left(x_{1}, \ldots, x_{n}\right)^{T}$ and $Y=\left(y_{1}, \ldots, y_{n}\right)^{T}$. The function $\phi(\cdot, \cdot): \mathbb{R} \times \mathbb{R} \rightarrow \mathbb{R}$ satisfies following three conditions. 1) $\phi(\cdot, \cdot)$ is a continuous mapping and satisfies the local Lipschitz condition.

2) There exists a positive constant $\alpha>0$, such that

$$
(x-y) \phi(y, x) \leq-\alpha(x-y)^{2} \forall x \neq y
$$

3) $\phi(x, y)=-\phi(y, x)$

Assumption 3: (H1) There exist constants $\delta_{i}$ such that $\left|f\left(x_{1}\right)-f\left(x_{2}\right)\right| \leq \delta_{i}\left|x_{1}-x_{2}\right|, i=1,2, \ldots, n, \forall x_{1}, x_{2} \in \mathbb{R}$.

(H2) Assume that the noise intensity function matrix $h(x(t))$ is uniformly Lipschitz continuous in terms of the norm induced by the trace inner product on the matrices, for all $\forall u \in \mathbb{R}^{n}$

$$
\operatorname{Tr}\left(h\left(u_{1}\right)-h\left(u_{2}\right)\right)^{T}\left(h\left(u_{1}\right)-h\left(u_{2}\right)\right) \leq \operatorname{Tr}\left[\beta\left\|u_{1}-u_{2}\right\|^{2}\right]
$$

where $\beta$ is a known constant.

Definition 1. The neural network system (3) is said to be synchronized if the trivial solution of system (5) satisfies

$$
\lim _{t \rightarrow+\infty}\left\|E\left(x_{i}(t)-s(t)\right)\right\|=0, \quad i=1,2, \ldots, N .
$$

Lemma 1. [37] Let $C^{2,1}\left(\mathbb{R}_{+} \times \mathbb{R}^{n} ; \mathbb{R}_{+}\right)$denote the family of all nonnegative functions $V(t)$ on $\mathbb{R}_{+} \times \mathbb{R}^{n}$, which are twice continuously differentiable in $x$ and once differentiable in $t$. If $V \in C^{2,1}\left(\mathbb{R}_{+} \times \mathbb{R}^{n} ; \mathbb{R}_{+}\right)$, define an operator $\mathcal{L} V$ from $\mathbb{R}_{+} \times \mathbb{R}^{n}$ to $\mathbb{R}$ by

$$
\mathcal{L} V(t)=V_{t}(t)+V_{x}(t) f(x(t))+\frac{1}{2} \operatorname{Tr}\left[\sigma(x(t))^{T} V_{x x}(t) \sigma(x(t))\right]
$$

where $V_{t}(t)=\partial V(t) / \partial t, V_{x}(t)=\left(\partial V(t) / \partial x_{1}, \ldots, \partial V(t) / \partial x_{n}\right), V_{x x}(t)=\left(\partial^{2} V(t) / \partial x_{i} x_{j}\right)_{n \times n}$. If $V \in C^{2,1}\left(\mathbb{R}_{+} \times \mathbb{R}^{n} ; \mathbb{R}_{+}\right)$, then for all $\infty>t>t_{0} \geq 0$,

$$
E V(t)=E V\left(t_{0}\right)+E \int_{t_{0}}^{t} \mathcal{L} V(s) d s
$$

as long as the expectations of the integrals exist. 


\section{Synchronization analysis}

\subsection{Synchronization with aperiodically intermittent control}

In this section, we will study the nonlinear coupled neural networks associated with stochastic perturbation via aperiodically intermittent control, and establish appropriate sufficient conditions such that the synchronization of the nonlinear coupled neural network (3) can be achieved.

Theorem 1: Suppose the Assumptions 1-3 hold and all the rest width $t_{i+1}-s_{i}$, for all $i=0,1,2, \ldots$ be bounded. If there exists diagnose matrix $P$ and positive constant $\varphi$ such that

$$
\lim _{i \rightarrow+\infty}\left[a_{1} \sum_{k=0}^{i}\left(s_{k}-t_{k}\right)+a_{2} \sum_{k=0}^{i}\left(t_{k+1}-s_{k}\right)\right]=-\infty
$$

where $a_{1}=\lambda_{\max }\left(-C-C^{T}+2 \beta^{2} I-2 \alpha(A+\Xi)\right)+\lambda_{\max }\left(\varphi \Delta P B+\frac{1}{\varphi} B^{T} P \Delta\right) P^{-1}$ and $a_{2}=\lambda_{\max }\left(-C-C^{T}+2 \beta^{2} I-2 \alpha A\right)+$ $\lambda_{\max }\left(\varphi \Delta P B+\frac{1}{\varphi} B^{T} P \Delta\right) P^{-1}, \Delta=\operatorname{diag}\left(\delta_{1}, \delta_{2}, \ldots, \delta_{n}\right)$, then the synchronization of the nonlinear coupled neural network with stochastic perturbation via aperiodically intermittent control (3) can be realized.

Proof: We consider the following standard Lyapunov function:

$$
V(t)=\sum_{i=1}^{N} e_{i}(t)^{T} P e_{i}(t)
$$

When $t \in\left[t_{i}, s_{i}\right)$, according to Lemma 1, we have

$$
\mathcal{L} V(t)=2 \sum_{i=1}^{N} e_{i}(t)^{T} P\left[-C e_{i}(t)+B g\left(e_{i}(t)\right)+\sum_{j=1, i \neq j}^{N} a_{i j} \phi\left(x_{j}(t), x_{i}(t)\right)+u_{i}(t)\right]+\sum_{i=1}^{N} \operatorname{Tr}\left[\tilde{\sigma}\left(e_{i}(t)\right)^{T} P \tilde{\sigma}\left(e_{i}\right)(t)\right]
$$

According to Assumption 3, we have

$$
\begin{array}{r}
2 \sum_{i=1}^{N} e_{i}(t)^{T} P B g\left(e_{i}(t)\right)=\sum_{i=1}^{N} e_{i}(t)^{T}\left[P B+B^{T} P\right] g\left(e_{i}(t)\right) \\
\leq \sum_{i=1}^{N} e_{i}(t)^{T}\left[\varphi \Delta P B+\frac{1}{\varphi} B^{T} P \Delta\right] e_{i}(t),
\end{array}
$$

while by Assumption 2, we have

$$
\begin{aligned}
& \sum_{i=1}^{N} e_{i}(t)^{T} P \sum_{j=1, i \neq j}^{N} a_{i j} \phi\left(x_{j}(t), x_{i}(t)\right)=\sum_{k=1}^{n} p_{k} \sum_{i=1}^{N} \sum_{j=1, i \neq j}^{N} a_{i j}\left[x_{i}^{k}(t)-s^{k}(t)\right] \phi\left(x_{j}^{k}(t), x_{i}^{k}(t)\right) \\
\leq & -\alpha \sum_{k=1}^{n} p_{k} \sum_{i=1}^{N} \sum_{j=1, i \neq j}^{N} a_{i j}\left[x_{j}^{k}(t)-x_{i}^{k}(t)\right]^{2}=-\alpha \sum_{i=1}^{N} \sum_{j=1, i \neq j}^{N} a_{i j}\left[x_{j}(t)-x_{i}(t)\right]^{T} P\left[x_{j}(t)-x_{i}(t)\right]=-\alpha e^{T}(t) A \otimes P e(t)
\end{aligned}
$$

and

$$
-\sum_{i=1}^{N} e_{i}(t)^{T} P \varepsilon_{i} \phi\left(x_{i}(t), s(t)\right) \leq-\alpha \sum_{i=1}^{N} e_{i}(t)^{T} P \varepsilon_{i} e_{i}(t)=-\alpha e^{T}(t) \Xi \otimes P e(t)
$$

Substituting (17)-(19) into (16) produces that

$$
\begin{aligned}
\mathcal{L} V(t) & \leq \sum_{i=1}^{N} e_{i}(t)^{T}\left[-P C-C^{T} P+2 \beta^{2} P\right] e_{i}(t)+2 \sum_{i=1}^{N} e_{i}(t)^{T}\left[\varphi \Delta P B+\frac{1}{\varphi} B^{T} P \Delta\right] e_{i}(t)^{T}-2 \alpha e^{T}(t) P \otimes(A+\Xi) e(t) \\
& \leq\left[\lambda_{\max }\left(-C-C^{T}+2 \beta^{2} I-2 \alpha(A+\Xi)\right)+\lambda_{\max }\left(\varphi \Delta P B+\frac{1}{\varphi} B^{T} P \Delta\right) P^{-1}\right] V(t)
\end{aligned}
$$


which implies

$$
E V(t) \leq E V\left(t_{i}\right) e^{a_{1}\left(t-t_{i}\right)}
$$

Similarly, when $t \in\left[s_{i}, t_{i+1}\right)$, we have

$$
\begin{aligned}
\mathcal{L} V(t) & \leq \sum_{i=1}^{N} e_{i}(t)^{T}\left[-P C-C^{T} P+2 \beta^{2} P\right] e_{i}(t)+\sum_{i=1}^{N} e_{i}(t)^{T}\left[\varphi \Delta P B+\frac{1}{\varphi} B^{T} P \Delta\right] e_{i}(t)^{T}-2 \alpha e^{T}(t) P \otimes A e(t) \\
& \leq\left[\lambda_{\max }\left(-C-C^{T}+2 \beta^{2} I-2 \alpha A\right)+\lambda_{\max }\left(\varphi \Delta P B+\frac{1}{\varphi} B^{T} P \Delta\right) P^{-1}\right] V(t)
\end{aligned}
$$

which implies

$$
E V(t) \leq E V\left(s_{i}\right) e^{a_{2}\left(t-s_{i}\right)}
$$

For $t \in\left[t_{0}, s_{0}\right)$, we have

$$
E V(t) \leq V\left(t_{0}\right) e^{a_{1}\left(t-t_{0}\right)}
$$

When $t \in\left[s_{0}, t_{1}\right)$, we have

$$
E V(t) \leq V\left(t_{0}\right) e^{a_{1}\left(s_{0}-t_{0}\right)+a_{2}\left(t-s_{0}\right)}
$$

For $t \in\left[t_{1}, s_{1}\right)$, we have

$$
E V(t) \leq E V\left(t_{1}\right) e^{a_{1}\left(t-t_{1}\right)} \leq V\left(t_{0}\right) e^{a_{1}\left(t-t_{1}\right)+a_{1}\left(s_{0}-t_{0}\right)+a_{2}\left(t_{1}-s_{0}\right)}
$$

When $t \in\left[s_{1}, t_{2}\right)$, we have

$$
E V(t) \leq E\left(s_{1}\right) e^{a_{2}\left(t-s_{1}\right)} \leq V\left(t_{0}\right) e^{a_{2}\left(t-s_{1}\right)+a_{1}\left(s_{1}-t_{1}\right)+a_{1}\left(s_{0}-t_{0}\right)+a_{2}\left(t_{1}-s_{0}\right)}=V\left(t_{0}\right) e^{a_{1} \sum_{k=0}^{1}\left(s_{k}-t_{k}\right)+a_{2}\left(t-s_{1}\right)+a_{2}\left(t_{1}-s_{0}\right)}
$$

For $t \in\left[t_{2}, s_{2}\right)$, we have

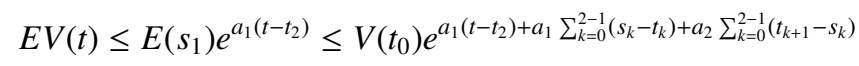

Similarly, we can prove that when $t \in\left[t_{i}, s_{i}\right)$, we have

$$
E V(t) \leq V\left(t_{0}\right) e^{a_{1}\left(t-t_{i}\right)+a_{1} \sum_{k=0}^{i-1}\left(s_{k}-t_{k}\right)+a_{2} \sum_{k=0}^{i-1}\left(t_{k+1}-s_{k}\right)}
$$

Thus when $t \in\left[s_{i}, t_{i+1}\right)$

$$
E V(t) \leq V\left(t_{0}\right) e^{a_{2}\left(t-s_{i}\right)+a_{1} \sum_{k=0}^{i}\left(s_{k}-t_{k}\right)+a_{2} \sum_{k=0}^{i-1}\left(t_{k+1}-s_{k}\right)}
$$

When $t=t_{i+1}$, we have

$$
E V\left(t_{i+1}\right) \leq V\left(t_{0}\right) e^{a_{1} \sum_{k=0}^{i}\left(s_{k}-t_{k}\right)+a_{2} \sum_{k=0}^{i}\left(t_{k+1}-s_{k}\right)}
$$

By combining all above estimates, we arrive at $\lim _{i \rightarrow+\infty} E V\left(t_{k}\right)=0$, which also implies $\lim _{t \rightarrow+\infty} E V\left(t_{k}\right)=0$ and $\lim _{t \rightarrow+\infty} E \| x_{i}(t)-$ $s(t) \|=0$, respectively. The proof is thus completed.

When $s_{i}-t_{i}=\theta$ and $t_{i+1}-s_{i}=\omega$, the control is periodically intermittent that is characterized by the following result.

Corollary 1: Suppose the Assumptions 1-3 hold. If there exists diagnose matrix $P$ and positive constant $\varphi$ such that

$$
a_{1} \theta+a_{2} \omega<0
$$

where $a_{1}$ and $a_{2}$ are defined in the Theorem 1 , then the nonlinear coupled complex network with stochastic perturbation via periodically intermittent control (3) will be synchronized. 


\subsection{Synchronization in terms of aperiodically adaptive intermittent control}

In the part, in order to realize synchronization of neural network by adaptive aperiodically intermittent control, the updating laws is set to be

$$
\dot{k}_{i}(t)= \begin{cases}\pi_{i} e^{c_{1} t}\left\|\phi\left(e_{i}(t)\right)\right\|^{2}, & t \in\left[t_{i}, s_{i}\right) \\ 0, & t \in\left[s_{i}, t_{i+1}\right)\end{cases}
$$

where $\pi_{i}$ and $c_{1}$ are positive constants, $k_{i}(0)>0, i=0,1, \ldots$ are initial value.

Then, based on the adaptive aperiodically intermittent controllers, the error systems can be written as

$$
\dot{x}_{i}(t)=\left[-C x_{i}(t)+B f\left(x_{i}(t)\right)+\sum_{j=1, i \neq j}^{N} a_{i j} \phi\left(x_{j}(t), x_{i}(t)\right)+k_{i}(t) u_{i}(t)\right] d t+\sigma\left(x_{i}(t)\right) d \omega(t) .
$$

Theorem 2: Suppose the Assumptions 1-3 hold and all the rest width $t_{i+1}-s_{i}, i=0,1,2, \ldots$ be bounded. If there exists a positive constant $\varphi$ such that

$$
\lim _{i \rightarrow+\infty}\left[\rho_{1} \sum_{k=0}^{i}\left(s_{k}-t_{k}\right)+\rho_{2} \sum_{k=0}^{i}\left(t_{k+1}-s_{k}\right)\right]=-\infty
$$

where $\left.\rho_{1}=\lambda_{\max }\left(-C-C^{T}+2 \beta^{2} I-2 \alpha A-2 k \alpha^{2} \Xi\right)\right)+\lambda_{\max }\left(\varphi \Delta B+\frac{1}{\varphi} B^{T} \Delta\right)$, and $\rho_{2}=\lambda_{\max }\left(-C-C^{T}+2 \beta^{2} I-2 \alpha A\right)+\lambda_{\max }\left(\varphi \Delta B+\frac{1}{\varphi} B^{T} \Delta\right)$, $\Delta=\operatorname{diag}\left(\delta_{1}, \delta_{2}, \ldots, \delta_{n}\right)$, then the synchronization of the nonlinear coupled neural network with stochastic perturbation via aperiodically intermittent control (3) can be realized.

Proof: Let's consider the following Lyapunov function:

$$
V(t)=\sum_{i=1}^{N}\left(e_{i}(t)^{T} e_{i}(t)+e^{-c_{1} t} \frac{\varepsilon_{i}\left(k_{i}(t)-k\right)^{2}}{\pi_{i}}\right)
$$

When $t \in\left[t_{i}, s_{i}\right)$, according to Lemma 1 , we have

$$
\begin{aligned}
\mathcal{L} V(t)= & 2 \sum_{i=1}^{N} e_{i}(t)^{T}\left[-C e_{i}(t)+B g\left(e_{i}(t)\right)+\sum_{j=1, i \neq j}^{N} a_{i j} \phi_{j}\left(x_{j}(t), x_{i}(t)\right)-\varepsilon_{i} k_{i}(t) \phi\left(x_{i}(t), s(t)\right)\right] \\
& -c_{1} \sum_{i=1}^{N} e^{-c_{1} t} \frac{\varepsilon_{i}\left(k_{i}(t)-k\right)^{2}}{\pi_{i}}+2 \sum_{i=1}^{N} \varepsilon_{i}\left(k_{i}(t)-k\right)\left\|\phi\left(e_{i}(t)\right)\right\|^{2} \dot{k}_{i}(t)+\sum_{i=1}^{N} \operatorname{Tr}\left[\tilde{\sigma}\left(e_{i}(t)\right)^{T} \tilde{\sigma}\left(e_{i}\right)(t)\right] \\
= & 2 \sum_{i=1}^{N} e_{i}(t)^{T}\left[-C e_{i}(t)+B g\left(e_{i}(t)\right)+\sum_{j=1, i \neq j}^{N} a_{i j} \phi_{j}\left(x_{j}(t), x_{i}(t)\right)\right] \\
& -c_{1} \sum_{i=1}^{N} e^{-c_{1} t} \frac{\varepsilon_{i}\left(k_{i}(t)-k\right)^{2}}{\pi_{i}}-2 \sum_{i=1}^{N} \varepsilon_{i} k\left\|\phi\left(e_{i}(t)\right)\right\|^{2}+\sum_{i=1}^{N} \operatorname{Tr}\left[\tilde{\sigma}\left(e_{i}(t)\right)^{T} \tilde{\sigma}\left(e_{i}\right)(t)\right] \\
\leq & \sum_{i=1}^{N} e_{i}(t)^{T}\left[-C-C^{T}+2 \beta^{2} I-2 \alpha A\right] e_{i}(t)+\sum_{i=1}^{N} e_{i}(t)^{T}\left[\varphi \Delta B+\frac{1}{\varphi} B^{T} \Delta\right] e_{i}(t)^{T} \\
& -c_{1} \sum_{i=1}^{N} e^{-c_{1} t} \frac{\varepsilon_{i}\left(k_{i}(t)-k\right)^{2}}{\pi_{i}}-2 \sum_{i=1}^{N} \varepsilon_{i} k\left\|\phi\left(e_{i}(t)\right)\right\|^{2}
\end{aligned}
$$

By Assumption 2, we get

$$
-\sum_{i=1}^{N} \varepsilon_{i} k\left\|\phi\left(x_{i}(t), s(t)\right)\right\|^{2} \leq-k \alpha^{2} \sum_{i=1}^{N} e_{i}^{T}(t) \varepsilon_{i} e_{i}(t)=-k \alpha^{2} \sum_{i=1}^{N} e^{T}(t) \Xi \otimes I e(t)
$$

Thus, we have

$$
\left.\mathcal{L} V(t) \leq\left[\lambda_{\max }\left(-C-C^{T}+2 \beta^{2} I-2 \alpha A-2 k \alpha^{2} \Xi\right)\right)+\lambda_{\max }\left(\varphi \Delta B+\frac{1}{\varphi} B^{T} \Delta\right)\right] V(t)
$$


which implies

$$
E V(t) \leq E V\left(t_{i}\right) e^{\rho_{1}\left(t-t_{i}\right)}
$$

Similarly, when $t \in\left[s_{i}, t_{i+1}\right)$, we have

$$
\begin{aligned}
\mathcal{L} V(t) & \leq \sum_{i=1}^{N} e_{i}(t)^{T}\left[-C-C^{T}+2 \beta^{2} I\right] e_{i}(t)+\sum_{i=1}^{N} e_{i}(t)^{T}\left[\varphi \Delta P B+\frac{1}{\varphi} B^{T} \Delta\right] e_{i}(t)^{T}-2 \alpha e^{T}(t) I \otimes A e(t) \\
& \leq\left[\lambda_{\max }\left(-C-C^{T}+2 \beta^{2} I-2 \alpha A\right)+\lambda_{\max }\left(\varphi \Delta B+\frac{1}{\varphi} B^{T} \Delta\right)\right] V(t)
\end{aligned}
$$

which implies

$$
E V(t) \leq E V\left(t_{i}\right) e^{\rho_{2}\left(t-s_{i}\right)}
$$

Define $W(t)=W(t, e(t))=e^{\gamma t} V(t)$, where $\gamma$ is the small positive constant. For $t \in\left[t_{i}, s_{i}\right)$, we calculate the operator

$$
\mathcal{L} W(t, e(t))=e^{\gamma t}[\gamma V(t)+\mathcal{L} V(t)] \leq e^{\gamma t}\left[\gamma V(t)+\rho_{1} V(t)\right]
$$

For $t \in\left[s_{i}, t_{i+1}\right)$, the operator is

$$
\mathcal{L} W(t, e(t))=e^{\gamma t}[\gamma V(t)+\mathcal{L} V(t)] \leq e^{\gamma t}\left[\gamma V(t)+\rho_{2} V(t)\right]
$$

Therefore, by the generalized Itô formula, we have that for any $t>t_{0} \geq 0$,

$$
W(t)=W\left(t_{0}\right)+E \int_{t_{0}}^{t} \mathcal{L} W(s) d s
$$

For $t \in\left[t_{0}, s_{0}\right)$, we have

$$
e^{\gamma t} E V(t) \leq E V\left(t_{0}\right)+\left(\gamma+\rho_{1}\right) \int_{t_{0}}^{t} e^{\gamma s} E V(s) d s \leq E V\left(t_{0}\right) e^{\left(\gamma+\rho_{1}\right)\left(t-t_{0}\right)}
$$

When $t \in\left[s_{0}, t_{1}\right)$, by using Gronwall inequality, we get

$$
\begin{array}{r}
e^{\gamma t} E V(t) \leq E V\left(s_{0}\right)+\left(\gamma+\rho_{2}\right) \int_{s_{0}}^{t} e^{\gamma s} E V(s) d s \leq E V\left(s_{0}\right) e^{\left(\gamma+\rho_{2}\right)\left(t-s_{0}\right)} \\
\leq E V\left(t_{0}\right) e^{\left(\gamma+\rho_{1}\right)\left(s_{0}-t_{0}\right)+\left(\gamma+\rho_{2}\right)\left(t-s_{0}\right)}
\end{array}
$$

For $t \in\left[t_{1}, s_{1}\right)$, we have

$$
e^{\gamma t} E V(t) \leq E V\left(t_{0}\right) e^{\left(\gamma+\rho_{1}\right)\left(s_{0}-t_{0}\right)+\left(\gamma+\rho_{2}\right)\left(t_{1}-s_{0}\right)+\left(\gamma+\rho_{1}\right)\left(t-t_{1}\right)}
$$

When $t \in\left[s_{1}, t_{2}\right)$, we obtain

$$
e^{\gamma t} E V(t) \leq E V\left(t_{0}\right) e^{\left(\gamma+\rho_{1}\right) \sum_{k=0}^{1}\left(s_{k}-t_{k}\right)+\left(\gamma+\rho_{2}\right)\left(t_{1}-s_{0}\right)+\left(\gamma+\rho_{2}\right)\left(t-s_{1}\right)}
$$

For $t \in\left[t_{i}, s_{i}\right)$, we have

$$
e^{\gamma t} E V(t) \leq E V\left(t_{0}\right) e^{\left(\gamma+\rho_{1}\right) \sum_{k=0}^{i-1}\left(s_{k}-t_{k}\right)+\left(\gamma+\rho_{2}\right) \sum_{k=0}^{i-1}\left(t_{k+1}-s_{k}\right)+\left(\gamma+\rho_{1}\right)\left(t-t_{i}\right)}
$$

When $t \in\left[s_{i}, t_{i+1}\right)$, we get

$$
e^{\gamma t} E V(t) \leq E V\left(t_{0}\right) e^{\left(\gamma+\rho_{1}\right) \sum_{k=0}^{i}\left(s_{k}-t_{k}\right)+\left(\gamma+\rho_{2}\right) \sum_{k=0}^{i-1}\left(t_{k+1}-s_{k}\right)+\left(\gamma+\rho_{2}\right)\left(t-s_{i}\right)}
$$

When $t=t_{i+1}$, we obtain

$$
\begin{aligned}
e^{\gamma t_{i+1}} E V(t) & \leq E V\left(t_{0}\right) e^{\left(\gamma+\rho_{1}\right) \sum_{k=0}^{i}\left(s_{k}-t_{k}\right)+\left(\gamma+\rho_{2}\right) \sum_{k=0}^{i}\left(t_{k+1}-s_{k}\right)} \\
& =E V\left(t_{0}\right) e^{\rho_{1} \sum_{k=0}^{i}\left(s_{k}-t_{k}\right)+\rho_{2} \sum_{k=0}^{i}\left(t_{k+1}-s_{k}\right)+t_{k+1}-t_{0}}
\end{aligned}
$$


which implies

$$
\begin{aligned}
E V(t) \leq & E V\left(t_{0}\right) e^{\left(\gamma+\rho_{1}\right) \sum_{k=0}^{i}\left(s_{k}-t_{k}\right)+\left(\gamma+\rho_{2}\right) \sum_{k=0}^{i}\left(t_{k+1}-s_{k}\right)} \\
& =e^{-\gamma t_{0}} E V\left(t_{0}\right) e^{\rho_{1} \sum_{k=0}^{i}\left(s_{k}-t_{k}\right)+\rho_{2} \sum_{k=0}^{i}\left(t_{k+1}-s_{k}\right)}
\end{aligned}
$$

Above estimates result in $\lim _{i \rightarrow+\infty} E V\left(t_{k}\right)=0$, which also indicates $\lim _{t \rightarrow+\infty} E V\left(t_{k}\right)=0$ and $\lim _{t \rightarrow+\infty} E\left\|x_{i}(t)-s(t)\right\|=0$. Therefore we complete the proof.

When $s_{i}-t_{i}=\theta$ and $t_{i+1}-s_{i}=\omega$, the control is periodically intermittent. We have the following result.

Corollary 2: Suppose the Assumptions 1-3 hold. If there exists a positive constant $\varphi$ such that

$$
\rho_{1} \theta+\rho_{2} \omega<0
$$

where $\rho_{1}$, and $\rho_{2}$ are defined in the Theorem 2 , then, the synchronization of the nonlinear coupled neural network with stochastic perturbation via periodically intermittent control (3) can be realized.

\section{Numerical example}

In this section, we provide numerical simulations of two examples to demonstrate the main results obtained in previous section.

Example 1: Consider the following neural networks:

$$
\dot{x}_{i}(t)=\left[-C x_{i}(t)+B f\left(x_{i}(t)\right)+\sum_{j=1, i \neq j}^{N} a_{i j}\left[\phi_{j}\left(x_{j}(t)\right)-\phi_{i}\left(x_{i}(t)\right)\right]\right] d t+\sigma\left(x_{i}(t)\right) d \omega(t)
$$

where $N=100, f(\cdot)=\frac{|x+1|-|x-1|}{2}, \phi(x(t))=x(t)+\tanh (x(t)), C=\operatorname{diag}(1.5,1.5), \beta=0.1$.

In this case, the coupling configuration matrix $A$ and the connection weight matrix $B$ are given by

$$
A=\left(\begin{array}{ccccc}
-1 & 1 & 0 & \cdots & 0 \\
0 & -1 & -1 & \cdots & 0 \\
\vdots & \vdots & \ddots & \ddots & \vdots \\
0 & \cdots & 0 & -1 & 1 \\
1 & 0 & \cdots & 0 & -1
\end{array}\right)_{100 \times 100} \quad B=\left(\begin{array}{cc}
2 & -0.1 \\
-5 & 4.5
\end{array}\right)
$$

Choose $P=\operatorname{diag}(1,1)$, and $\Xi=\operatorname{diag}(3,3)$, and we have $\alpha=3.5, \varphi=1$. Let the aperiodically intermittent control parameters be $\theta=0.1$ and $\omega=0.6$. Through a direct computation, we have $a_{1}=-16.49$ and $a_{2}=4.51$. Thus, as $t \rightarrow+\infty$, one can see $\left[a_{1} \sum_{k=0}^{i}\left(s_{k}-t_{k}\right)+a_{2} \sum_{k=0}^{i}\left(t_{k}-s_{k}\right)\right]=-\infty$. Therefore the error dynamics of the neural networks (55) can be stabilized and it is consistent with the result of Theorem 1.

Figure 1 depicts the trajectories of error states of (55) without aperiodically intermittent, which indicates that the network (55) without aperiodically intermittent cannot synchronize itself. Figure 2 shows the trajectories of error states of the system with aperiodically intermittent, which approach to zero as time increases. Hence the network is synchronized under the synchronizing aperiodically intermittent control.

Example 2: In the following example, we consider the neural networks:

$$
\dot{x}_{i}(t)=\left[-C x_{i}(t)+B f\left(x_{i}(t)\right)+\sum_{j=1, i \neq j}^{N} a_{i j} \phi\left(x_{j}(t), x_{i}(t)\right)+h_{i}(t) u_{i}(t)\right] d t+\sigma\left(x_{i}(t)\right) d \omega(t)
$$

where $h_{i}(t)=\pi_{i} e^{-\rho_{1} t}\left\|\phi\left(e_{i}(t)\right)\right\|_{2}^{2}, \phi=3, C=\operatorname{diag}(2.5,2.5)$ and $N=100$. The other parameters are the same as those given in Example 1.

By a simple computation, we have $\rho_{1}=-19.54$ and $\rho_{2}=2.51$. Similar to Example 1, one can see that the error dynamics of this neural networks (55) is asymptotically stable, as predicted by Theorem 2. Figure. 3 and Figure. 4 show the synchronization errors of $x_{11}-x_{i 1}$ and $x_{12}-x_{i 2}$, respectively. These figures indicate that synchronization can be achieved. 


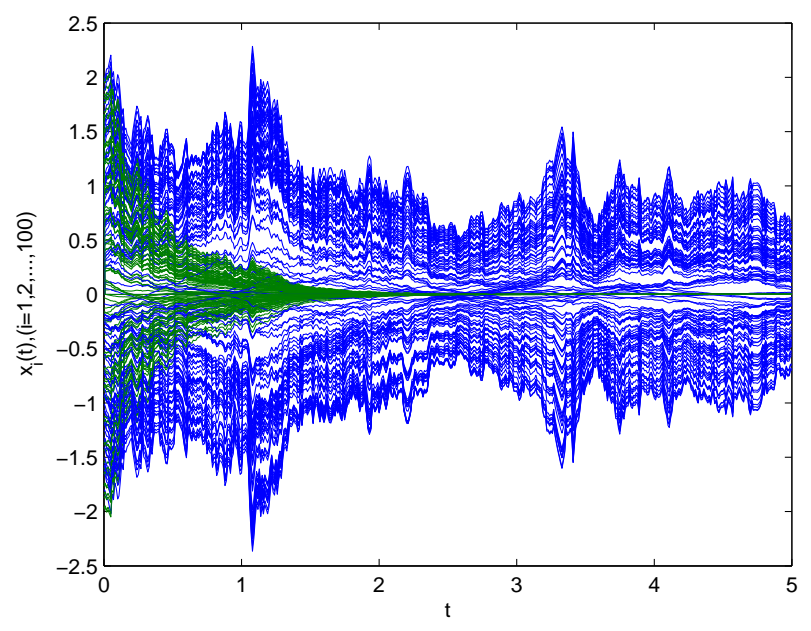

Figure 1: Time response of the error states of (55) without aperiodically intermittent

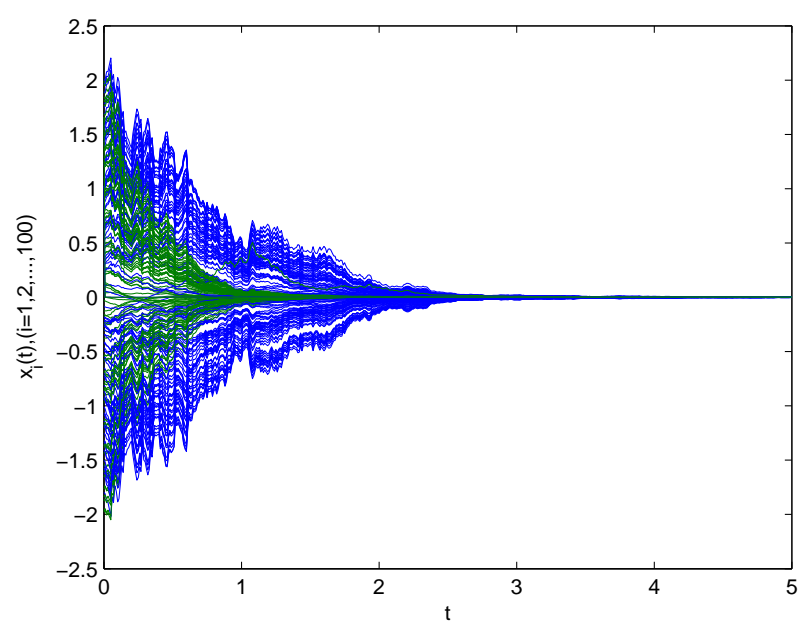

Figure 2: Time response of the error states (55) with aperiodically intermittent

\section{Conclusions}

Periodically intermittent control for neural networks may not be realistic in many real applications, in particular, when uncertainties are taken into the consideration. In this paper, we study the synchronization problem for neural networks with stochastic perturbation via aperiodically intermittent control as well as adaptive aperiodically intermittent control. Under the framework of stochastic theory and Lyapunov stability method, sufficient conditions are derived to guarantee the synchronization by making use of the adaptive aperiodicity intermittent control technique. Numerical simulations demonstrate the effectiveness of the proposed approach. Our next goal is to investigate the synchronization for neural networks with time-varying delays via aperiodically intermittent control, and the results will be reported elsewhere.

\section{Acknowledgment}

This publication was made possible by NPRP grant $\sharp$ NPRP 4-1162-1-181 from the Qatar National Research Fund (a member of Qatar Foundation). The statements made herein are solely the responsibility of the authors. This work was also supported in part by Natural Science Foundation of China (grant nos: 61374078, 61403313, 61403050), and in part by NSF 1021203, 1419028 of the United States. 


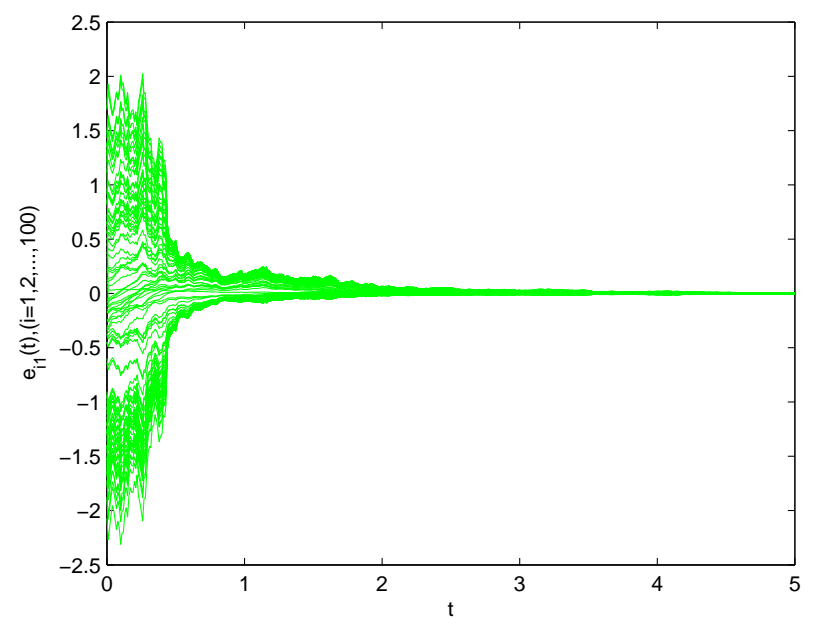

Figure 3: Time response of the error states of (56) with adaptive aperiodically intermittent

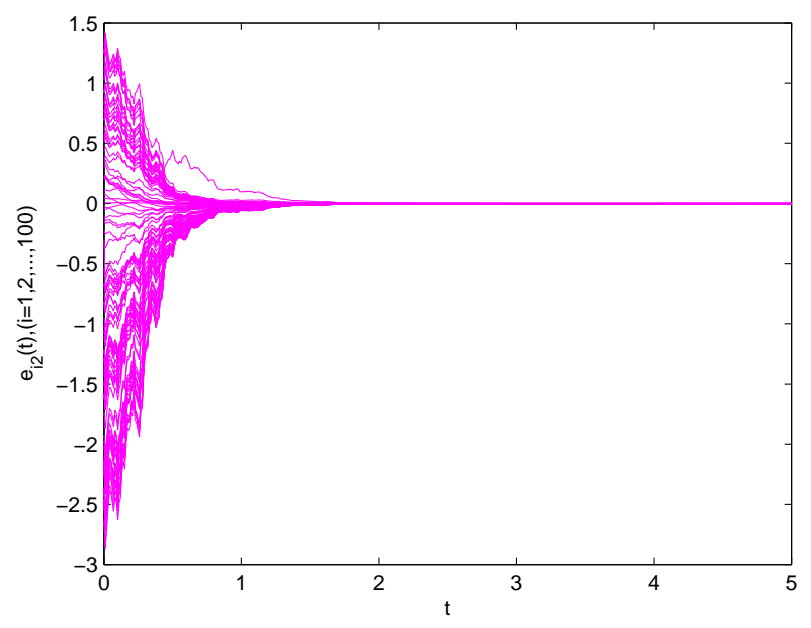

Figure 4: Time response of the error states (56) with adaptive aperiodically intermittent

\section{References}

[1] Watts, D., \& Strogatz, S.(1998). Collective dynamics of 'small-word' networks. Nature, 393(6684), 440-442.

[2] Barabasi, A., \& Albert, R. (1999) Emergence of scaling in random networks. Science, 286(5439), 509-512.

[3] Guan, Z., Liu, Z., Feng, G., \& Wang, Y.(2010) Synchronization of complex dynamical networks with time-varying delays via impulsive distributed control. IEEE Transactions on networks and learning systems, 57(8), 2182-2195.

[4] Li, C., Yu, W., \& Huang, T. (2014). Impulsive synchronization schemes of stochastic complex networks with switching topology: average time approach. Neural Networks, 54, 85-94.

[5] He, X., Li, C., Huang, T., \& Li, C. (2014). Neural network for solving convex quadratic bilevel programming. Neural Networks, $51(3), 17-25$.

[6] Wen, S., Huang, T., \& Zeng, Z. (2015). Circuit design and exponential stabilization of memristive neural networks. Neural Networks, 63, 48-56.

[7] Lu, J., Ho, D., \& Wang, Z. (2009). Pinning stabilization of linearly coupled stochastic neural networks via minimum number of controllers. Neural Networks, IEEE Transactions on, 20(10), 1617-1629.

[8] He, X, Yu, J., Huang, T., Li, C., \& Li, C. (2014). Neural networks for solving Nash Equilibrium problem in application of multiuser power control. Neural Networks, 57(9), 73-78.

[9] Wen, P., Zeng, Z., \& Huang, T. Exponential lag adaptive synchronization of memristive neural networks and applications in Pseudo-random generators. IEEE Transactions on Fuzzy Systems, DOI: 10.1109/TFUZZ.2013.2294855.

[10] Liu, Y., Wang, Z., Liang, J., \& Liu, X. (2009) Stability and synchronization of discrete-time Markovian jumping neural networks with mixed modedependent time delays. IEEE Trans. Neural networks, 20(7), 1102-1116. 
[11] He, X, Li, C., Huang, T., Li, C., \& Huang, J. (2014). A Recurrent Neural Network for Solving Bilevel Linear Programming Problem. IEEE Transactions on Neural Networks and Learning Systems, 25(4), 824-830.

[12] Wen, S., Bao, G., \& Zeng, Z. (2013). Global exponential synchronization of memristor-based recurrent neural networks with time-varying delays Neural Networks, 48, 195-203.

[13] Arenas, A., Guilera, A., Kurths, J., Morenob, Y., \& Zhoug, C. (2008). Synchronization in complex networks, Phys Rep, 469(3),93-153.

[14] Tang, Y., Wang, Z., Gao, H., Swift, S., \& Kurths, J. (2012). A constrained evolutionary computation method for detecting controlling regions of cortical networks. IEEE/ACM Tran. Comput. Bol. Bioinf, 9(6), 1569-1581.

[15] Strogatz, S., \& Stewart, I. (1993). Coupled oscollators and biological synchronization. Sci Am, 269(6),102-9.

[16] Wu,C. (2007). Synchronization in complex networks of nonlinear dynamical systems. World Scientfic, singapore.

[17] Huang, H., Ho, D., \& Cao, J. (2005). Analysis of global exponential stability and periodic solutions of neural networks with time-varying delays. Neural Networks, 18(2), 161-170.

[18] Zhang, Y., \& Chen, G. (2009). Fuzzy impulsive control of chaotic systems based on TS fuzzy model. Chaos, Solitons Fractals 39(4), 2002-2011.

[19] Gao, Q., Feng, G., \& Xi, Z. (2014). A new design of robust h sliding mode control for uncertain stochasti T-S fuzzy time-delay systems. IEEE transactions on cybernetics, 44(9), 1556-66.

[20] Yang, X., Cao, j., \& Lu, J. (2011). Synchronization of delayed complex dynamical networks with impulsive and stochastic effects. Nonlinear Analysis: Real World Application, 12(4), 2252-2266.

[21] Li, C., Li, C., \& Liao, X. (2011). Impulsive effects on stability of high-order BAM neural networks with time delays. Neurocomputing, 74(10), $1541-1550$

[22] Li, X. \& Song, S. (2013) Impulsive control for existence, uniqueness andglobal stability of periodic solutions of recurrentneural networks with discrete and continuouslydistributed delays. IEEE Transactions on Neural Networks and Learning Systems, 24(6), 868-877.

[23] Zhang, W., Tang, Y., Miao, Q., \& Du, W. (2013). Exponential synchronization of coupled switched neural networks with mode-dependent impulsive effects. IEEE Transactions on networks and learning systems, 24(8), 1316-1326.

[24] $\mathrm{Hu}, \mathrm{C} .$, Yu, J., \& Jiang, H., Exponential stabilization and synchronization of neural networks with time-varying delays via periodically intermittent control. Nonlinearity, 23(10), 2369.

[25] Zochowski, M. (2000). Intermittent dynamical control. Physica D, 145(3), 181-190.

[26] Cai, S., Liu, Z., Xu, F., \& Shen, J. (2009). Periodically intermittent controlling complex dynamical networks with time-varying delays to a desired orbit. Physics Letter A, 373(42), 3846-3854.

[27] Yu, J., Hu, C., Jiang, H., \& Teng, Z. (2012). Exponential lay synchronization for delayed fuzzy cellular nerual networks via periodically intermittent control. Mathematics and Computers in Simulation, 82(5), 895-908.

[28] Xia, W., \& Cao, J. (2009). Pinning synchronization of delayed dynamical networks via periodically intermittent control. Chaos, 19(1), 013120.

[29] Wang, J., Feng, J., Xu, C., \& Zhao, Y. (2013). Exponential synchronization of stochastic perturbed complex networks with time-varying delays via periodically intermittent pinning. Commun Nonlinear Sci Numer Simulat, 18(11), 3146-3157.

[30] Huang, H., Feng, G., \& Cao, J. (2008). Robust state estimation for uncertain neural networks with time-varying delay. Neural Networks, IEEE Transactions on, 19(8), 1329-1339.

[31] Lu, J., Ho, D., \& Cao, J. (2008). Synchronization in an array of nonlinearly coupled chaotic neural networks with delay coupling. International Journal of Bifurcation and Chaos, 18(10), 3101-3111.

[32] Yang, X., Cao, J. (2009). Stochastic synchronization of coupled neural networks with intermittent control. Physics Letters A, $373(36), 3259-3272$.

[33] Pototsky, A., \& Janson, N. (2009). Synchronization of a large number of continuous one-dimensional stochastic elements with time-delayed mean-field coupling. Physica D, 238(2), 175-183.

[34] Liu, X, Chen, T. (2014). Synchronization of nonlinear coupled networks via aperiodically intermittent pinning control. IEEE Transactions on Neural Networks and Learning Systems, 2162-237x.

[35] Lu, W., \& Chen, T. (2006). New approach to synchronization analysis of linearly coupled ordinary differential systems. Phy. D, Nonlinear Phenomena, 213(2), 214-230.

[36] Liu, X., \& Chen, T. (2008). Synchronization analysis for nonlinear-coupled complex networks with an asymmetrical coupling matrix. Phy. A, Statist. Mech. Appl, 387(16), 4429-4439.

[37] Yuan, C., \& Mao, X. (2004). Robust stability and controllability of stochastic differential delay equations with markovian switching. Automatica, 40(3), 343-54. 